\title{
Bilayer Beams and Relay Sharing based OFDMA Cellular Architecture
}

\author{
Yanxiong Pan ${ }^{1,2}$ \\ ${ }^{1}$ University of Science \& Technology of China/Dept. of Electronic Engineering \& Information Science, Hefei, China \\ ${ }^{2}$ Xichang Satellite Launch Center/Yibin Tracking Telemetry Station, Yibin, China \\ Email: yxpan8@mail.ustc.edu.cn
}

Hui $\mathrm{Han}^{3}$, Sihai Zhang ${ }^{1}$ and Wuyang Zhou ${ }^{1}$

${ }^{3}$ Chongqing Communication Institute of P.L.A/Dept. of Information Engineering, Chongqing, China

Email: haipiao@mail.ustc.edu.cn; \{shzhang,wyzhou\}@ustc.edu.cn

\begin{abstract}
Over the past decade, researchers have been putting a lot of energy on co-channel interference suppression in the forthcoming fourth generation (4G) wireless networks. Existing approaches to interference suppression are mainly based on signal processing, cooperative communication or coordination techniques. Though good performance has been attained already, a more complex receiver is needed, and there is still room for improvement through other ways.

Considering spatial frequency reuse, which provides an easier way to cope with the co-channel interference, this paper proposed a bilayer beams and relay sharing based (BBRS) OFDMA cellular architecture and corresponding frequency planning scheme. The main features of the novel architecture are as follows. Firstly, the base station (BS) uses two beams, one composed of six wide beams providing coverage to mobile stations (MSs) that access to the BS, and the other composed of six narrow beams communicating with fixed relay stations (FRSs). Secondly, in the corresponding frequency planning scheme, soft frequency reuse is considered on all FRSs further. System-level simulation results demonstrate that better coverage performance is obtained and the mean data rate of MSs near the cell edge is improved significantly. The BBRS cellular architecture provides a practical method to interference suppression in 4G networks since a better tradeoff between performance and complexity is achieved.
\end{abstract}

Index Terms_-interference, relay, OFDMA, cellular system, frequency planning

\section{INTRODUCTION}

Orthogonal frequency division multiplexing (OFDM) technique has high spectral efficiency and inherit immunity to frequency selective fading, therefore the

Manuscript received April xx, 2011; revised April xx, 2011; accepted April xx, 2011.

This work is supported by the China High-Tech 863 Plan under Grant 2009AA011506, the National Major Special Projects in Science and Technology of China under Grant 2009ZX03003-009, 2010ZX03003-001, and 2010ZX03005-003, the National Key Technology R\&D Program under Grant 2008BAH30B12, and the National Basic Research Program of China under Grant 2007CB310602. corresponding multiplexing technique orthogonal frequency division multiple access (OFDMA) has already become one of the key techniques of the candidates for the next generation (4G) mobile communication standards, such as 3GPP LTE-Advanced [1] and IEEE 802.16j [2]. In OFDM technique, the total system bandwidth is divided into several sub-carriers, and the spectra of two adjacent sub-carriers have $50 \%$ overlap with each other, resulting in higher spectral efficiency compared with traditional frequency division multiplexing. When the bandwidth of each sub-carrier is smaller than the coherence bandwidth of the channel, the frequency selective fading channel can be converted into flat fading channel. Under the protection of the cyclic prefix, the inter symbol interference (ISI) resulted from multipath propagation is reduced significantly, thus better communication quality is achieved.

One of the basic features of $4 \mathrm{G}$ wireless network is broadband. According to the definition of the International Telecommunication Union (ITU), 4G network must achieve a data rate higher than 100Mbps in the downlink, which urges to increase the utilization efficiency of the limited spectrum resource as much as possible. The extreme way called unitary frequency reuse, in which each cell uses the whole system bandwidth, can make better use of the spectrum resource, but may cause severe co-channel interference to cell edge users. If unitary frequency reuse cannot meet the system capacity requirement, sectorization technique, in which the omnidirectional antenna at the base station (BS) is replaced by several sector antennas, is usually used. However, if aggressive frequency reuse with each sector uses the whole system bandwidth is adopted, and no effective interference suppression measures are used, the interference will be even more unbearable.

It is a very important way to enhance network performance by using special fixed relay stations (FRSs) to forward data to and thus boosting the performance of cell edge users [3]. However, the introduction of FRSs brings new challenges to frequency planning, thus more effort on interference suppression is needed. 


\section{A. Traditional Approaches to Interference Suppression}

Interference suppression in OFDMA networks has been a hot topic in recent years, with lots of effective methods being proposed, but most of them need a high complex receiver. Three ways to interference suppression are considered in 3GPP LTE system, i.e., interference randomization, interference cancellation and interference coordination/mitigation [1,4]. Cell specific scramble codes, interleaving and frequency hopping techniques are used in interference randomization, but the interference power is only randomized over the whole system bandwidth and not actually decreased. Interference cancellation is based on interference detection and subtraction, and high complexity makes it usually been implemented at the base stations (BSs). However, in interference coordination/mitigation, restrictions on resource allocation between cells are considered. Though better Carrier to Interference plus Noise Ratio (CINR) is attained, it causes higher signaling overhead.

\section{B. Frequency Planning Techinique}

Careful frequency planning can suppress interference efficiently. A factor $R_{I}$, which describes the ratio of interference from all BSs to that from all FRSs in downlink access zone (AZ), is introduced to model the interference status of each mobile station (MS) in [5]. According to different $R_{I}$, the total system bandwidth is divided into three subsets, the first two of which can be used only by BS and FRS, respectively, and the other is used by BS and FRS simultaneously. It is a dynamic frequency planning scheme, in which better performance is achieved. But each BS sector uses the whole system bandwidth with no interference suppression strategies in downlink relay zone (RZ), leading to severe co-channel interference. Two adaptive frequency planning schemes are proposed in [6]. A better compromise between spectral efficiency and interference is achieved through frequency reuse factor (FRF) adaptation in different time zones. However, each BS sector can only use one third of the total system bandwidth in downlink AZ, resulting in lower system capacity.

\section{Sectorization Techinique}

The sectorization technique, in which each cell is covered by several sector antennas, is not only an approach to higher system capacity, but also a good method for interference suppression. Sector antenna only transmits radio signal to the directions concerned, thus the interference to other directions is reduced. However, in most previous works [5-6], relay stations are equipped with omnidirectional antennas, causing interference to stations transmitting on the same channel in all directions around. Furthermore, relay stations are usually placed in each BS sector, leading to higher costs when the network is larger.

A sector relay based cell architecture is proposed in [7]. Being able to communicate with the three nearest BSs simultaneously, FRSs equipped with three sector antennas are located on common vertices of hexagonal cells, thus the number of FRSs needed is dropped. But in each cell, the total system bandwidth is partitioned into seven parts, only one of which can be used by the BS and each FRS, respectively. Similarly, Lee et al proposed a shared relay segmentation (SRS) cell architecture [8]. Each BS and FRS has three sector antennas, using the whole and one third of the total system bandwidth, respectively. FRSs only located on those common vertices that BS antennas pointed to. Though cooperative communication between BS and FRS is adopted and no interference needs to be considered in the same BS sector, the interference between adjacent BS sectors in the same cell is still severe. An ideal sector antenna model, the border of which is a straight line, is used in [9], resulting in zero signal strength in some directions. In practice, a sector antenna radiates signal to all directions around it with different gains. Therefore, when the number of interfering stations is big, large errors will appear in the performance analysis.

In this paper, a bilayer beams and relay sharing based (BBRS) OFDMA cellular architecture and corresponding frequency planning scheme are proposed. Our contribution is threefold. Firstly, a novel architecture is designed. The two beam layers generated by sector antennas on each BS can be viewed as one type of space division multiplexing (SDM), which brings down the co-channel interference of aggressive frequency reuse and increases the mean data rate of cell edge users with that of cell central users guaranteed. Secondly, a static frequency planning scheme that compatible with the characteristics of the BBRS architecture is proposed. Each BS sector can use one half of the total system bandwidth. Furthermore, the soft frequency reuse on FRSs increases the mean data rate of cell edge users and system throughput, so the spectral efficiency is raised. Thirdly, an in-depth analysis of system performance and numerous simulations are carried out, which provides a reference for actual system design. Simulation results illustrate that BBRS architecture achieves a better compromise between performance and complexity.

The rest of the article is organized as follows. The BBRS architecture and corresponding frequency planning scheme are introduced in section II. The CINR performance is analyzed in section III. Rate mapping strategy, path selection and scheduling algorithm are elaborated in section IV. Simulation results are presented in section V. At last, we conclude this article in section VI.

\section{BBRS ARCHITECTURE}

\section{A. System Model and the BBRS Architecture}

The performance of the central cell, i.e., Cell 1 , is evaluated in a 19-cell OFDMA network, cells marked as (2) to (19) are interfering cells, as shown in Fig. 1. Each cell is divided into $S_{T}$ sectors with $M$ MSs uniformly distributing in each sector, only the handoff in each BS sector is considered throughout this paper. The frame structure for non-transparent relay stations in IEEE 802.16j [2] is adopted, as shown in Fig. 2. We only consider the downlink performance, with $\mathrm{AZ}$ and $\mathrm{RZ}$ representing the access zone and relay zone in downlink sub-frame. Unitary frequency reuse is employed, and each BS has the same frequency planning. The system works 


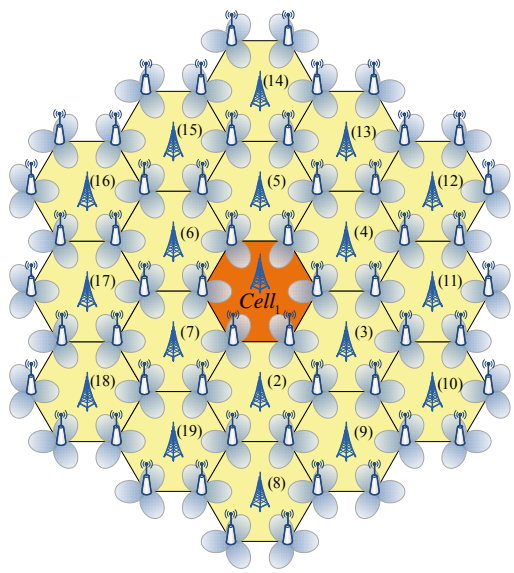

Figure 1. 19-cell BBRS cellular network.

under Time Division Duplexing (TDD) with perfect time synchronization.

In the BBRS architecture, each cell is partitioned into six sectors. BS is located in cell center while FRSs, the total number of which in the 19-cell network is 54, are placed on common vertices of the hexagonal cells. Each FRS works under decode-and-forward (DF) mode, and can communicate with three adjacent BSs simultaneously, since equipped with three sector antennas. The total system bandwidth, i.e., $B W$, is divided into $N$ sub-channels, each of which consists of $C$ adjacent subcarriers. $N$ sub-channels are further divided into 6 orthogonal subsets in the same size, i.e. $\left\{r_{i}|| r_{i} \mid=N / 6, i=1,2, \ldots, 6\right\}$.

The SRS architecture and corresponding frequency planning scheme [8] is shown as Fig. 3. There are only three FRSs in each cell, so the total number of FRSs in the 19-cell network will be 27 .

\section{B. Frequency Planning Scheme in BBRS Architeture}

In the BBRS system, the main lobe pattern of the antennas and the frequency planning scheme are depicted as Fig. 4 and Fig. 5, respectively. Each BS sector has two beams, one of which is wider and used for providing coverage to cell center MSs, the other is narrower and used for communicating with the nearest FRS.

\section{1) Frequency Planning at Base Stations}

Consider the base station in the central cell, i.e., $B S_{1}$. In

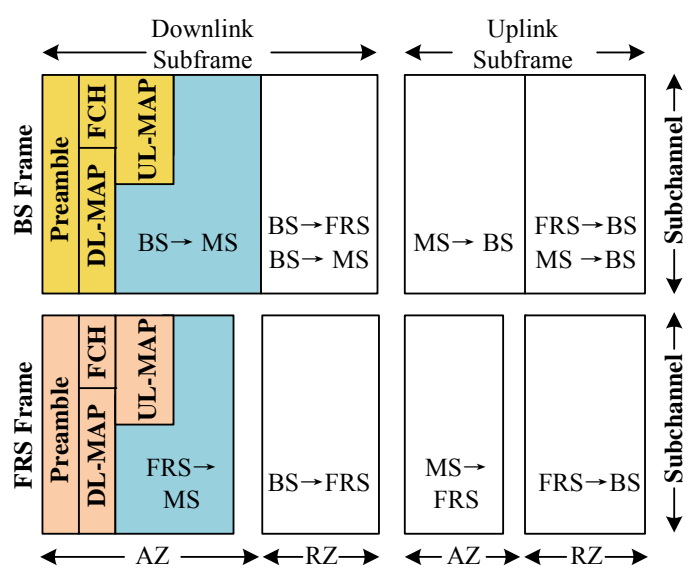

Figure 2. Frame structure of IEEE 802.16j.

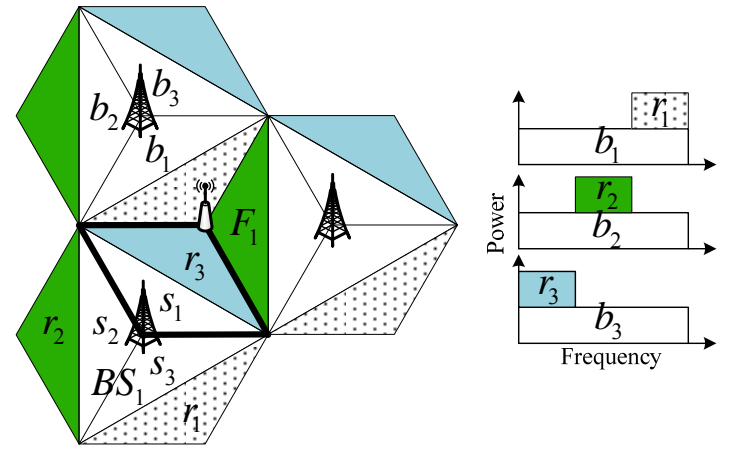

Figure 3. SRS cell architecture and frequency planning.

AZ, only wide beams are active. Odd sectors, i.e., $s_{1}, s_{3}$ and $s_{5}$, reuse sub-channel set $b_{1}=\left\{r_{1}, r_{2}, r_{3}\right\}$, and even sectors, i.e., $s_{2}, s_{4}$ and $s_{6}$ reuse $b_{2}=\left\{r_{4}, r_{5}, r_{6}\right\}$, as shown in Fig. 4(a). In RZ, both wide and narrow beams are turned on. The frequency planning of wide beams is unchanged, and that of narrow beams is as follows. Odd sectors $s_{1}, s_{3}$ and $s_{5}$ reuse $b_{2}=\left\{r_{4}, r_{5}, r_{6}\right\}$, and even sectors $s_{2}, s_{4}$ and $s_{6}$ reuse $b_{1}=\left\{r_{1}, r_{2}, r_{3}\right\}$, as shown in Fig. 4(b).

\section{2) Frequency Planning at Relay Stations}

All FRSs can be classified into two categories, i.e., odd relays and even relays, shared by odd sectors and even sectors of BSs, with $F_{1}$ and $F_{2}$ as the representatives, respectively, as illustrated in Fig. 4. FRSs of the same category have the same frequency planning.

Relay stations do not transmit in RZ, so only the frequency planning in AZ needs to be considered. As shown in Fig. 4(a), each FRS uses sub-channels that orthogonal with adjacent BS sectors, thus $F_{1}$ uses $b_{2}$ and $F_{2}$ uses $b_{1}$. In order to reduce the interference to cell edge users, available sub-channels on each FRS is divided into three parts in the same size, i.e., N/6 sub-channels, and assigned to each sector.

Soft Frequency Reuse (SFR) employs zone-based reuse factors in the cell center and the cell edge areas [10]. Center areas of all cells use the same band with lower transmit power, achieving an efficient use of spectrum resource. Near the cell edge, the sub-channels allocated to adjacent cells are orthogonal and have higher transmit power, so as to control the co-channel interference and guarantee the coverage performance. Inspired by this idea, in order to provide more available sub-channels to relay users, we apply SFR on all FRSs as follows. On the basis of existing frequency planning, each FRS further reuses sub-channels of the three nearest BS sectors with lower

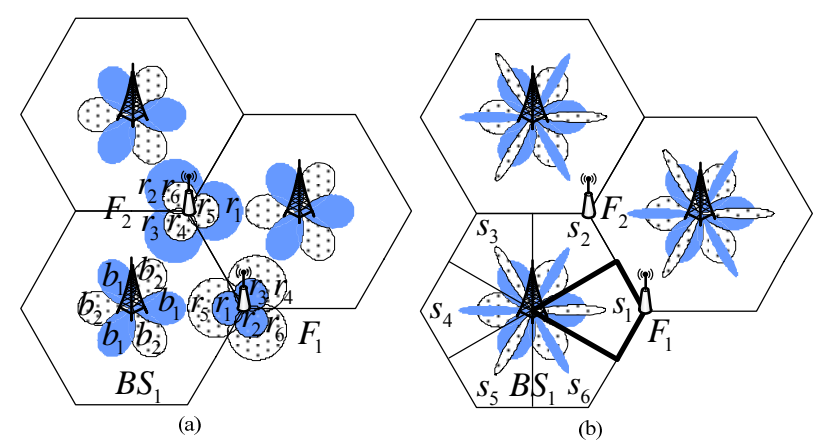

Figure 4. Main lobe pattern of antennas in BBRS system. 

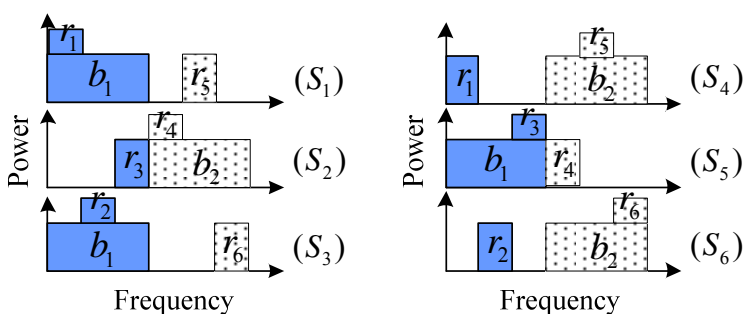

Figure 5. Frequency planning scheme in BBRS system.

transmit power, i.e., $F_{1}$ reuses $b_{1}$ and $F_{2}$ reuses $b_{2}$. Therefore, the number of available sub-channels on each FRS sector reaches $N / 3$, and higher cell edge throughput can be expected.

Finally, the frequency planning at FRSs can be described as follows: three sectors of $F_{1}$ use $\left\{r_{3}, r_{4}\right\},\left\{r_{1}, r_{5}\right\}$ and $\left\{r_{2}, r_{6}\right\}$, and $F_{2}$ allocates $\left\{r_{5}, r_{1}\right\},\left\{r_{6}, r_{2}\right\}$ and $\left\{r_{4}, r_{3}\right\}$ to each sector. In each sub-channel set, the transmit power of the two elements are $P_{\text {low }}$ and $P_{h}$, respectively, with $P_{\text {low }} \leq P_{h}$ and the power ratio $\rho=P_{\text {low }} / P_{h}$.

\section{CINR PERFORMANCE ANALYSIS}

Assuming all the buffers of MSs are full, all available sub-channels on BSs and FRSs are used up. Interference is in its worst case, and the system performance reaches the lower bound. Equal power allocation strategy is employed, in which the total sector power of BS and FRS is allocated equally to each sub-carrier.

The CINR of the BBRS system will be analyzed first. Only the handoff in each BS sectors is considered, so the analysis of one sector of Cell ${ }_{1}$ is enough. In this section, all analyses are based on sector $s_{1}$ in Cell $_{1}$, which is the area with bold border in Fig. 4(b). According to certain path selection strategy, MSs access to either $B S_{1}$ or $F_{1}$, with corresponding user sets are $\mathbf{D}=\left\{d_{m}, m=1,2, \ldots, M_{1}\right\}$ and $\mathbf{Q}=\left\{q_{n}, n=1,2, \ldots, M_{2}\right\}$, where $M_{1}+M_{2}=M$.

\section{A. Interference Analysis in AZ}

In AZ, user $d_{m}$ in set $\mathbf{D}$ suffers interference from all BSs and FRSs. In the BBRS system, $N$ sub-channels are reused three times and once on BS and FRS, respectively. Average power values of useful signal and the interference from BSs and from FRSs on sub-channel $k$ of $d_{m}$ are:

$$
\begin{gathered}
P_{r}=P_{B} \cdot G\left(1,1, d_{m}, k\right) \cdot A_{T}\left(1,1, d_{m}\right), \\
P_{I B S}=\sum_{s \in\{1,3,5\}} \sum_{b=1}^{19}\left[P_{B} \cdot G\left(b, s, d_{m}, k\right) \cdot A_{T}\left(b, s, d_{m}\right)\right]-P_{r} \\
P_{I F R S}=\sum_{f=1}^{N_{r}}\left[P_{F} \cdot G\left(f, d_{m}, k\right) \cdot A_{T}\left(f, d_{m}\right)\right],
\end{gathered}
$$

where $N_{r}, P_{B}$ and $P_{F}$ are the total number of FRSs in the system, the transmit power on each subcarrier of BS and FRS, respectively. $G\left(b, s, d_{m}, k\right)$ and $G\left(f, d_{m}, k\right)$ are the channel gains from sector $s$ of $B S_{\mathrm{b}}$ and $F R S_{\mathrm{f}}$ to $d_{m}$, with $A_{T}\left(b, s, d_{m}\right)$ and $A_{T}\left(f, d_{m}\right)$ being the antenna gains.
User $q_{n}$ in set $\mathbf{Q}$ suffers co-channel interference from all BSs and all FRSs except $F_{1}$. Mean power values of useful signal and the interference from BSs and FRSs on sub-channel $k$ of $q_{n}$ are:

$$
\begin{gathered}
P_{r}=P_{F} \cdot G\left(1, q_{n}, k\right) \cdot A_{T}\left(1, q_{n}\right), \\
P_{I B S}=\sum_{\substack{s \in\{1,3,5\} \\
\text { or }\{2,4,6\}}}^{19}\left[P_{B} \cdot G\left(b, s, q_{n}, k\right) \cdot A_{T}\left(b, s, q_{n}\right)\right], \\
P_{I F R S}=\sum_{f=2}^{N_{r}}\left[P_{F} \cdot G\left(f, q_{n}, k\right) \cdot A_{T}\left(f, q_{n}\right)\right] .
\end{gathered}
$$

The variable $s$ that stands for co-channel sectors in each cell in (5) will be chosen from $\{1,3,5\}$ if $k \in b_{1}$, otherwise it will be chosen from $\{2,4,6\}$.

\section{B. Interference Analysis in $R Z$}

In $R Z$, only relay station $F_{1}$ and users in $\mathbf{D}$ are receiving from $B S_{1}$. The useful signal of $F_{1}$ comes from the narrow beam of $s_{1}$, and the interference comes from all BSs. Mean power of useful signal on sub-channel $k$ of $F_{1}$ is:

$$
P_{r}=P_{B} \cdot G_{n}\left(1,1, F_{1}, k\right) \cdot A_{T n}\left(1,1, F_{1}\right) \cdot A_{R}\left(1,1, F_{1}\right) .
$$

The average power of interference from wide and narrow beams of all BSs to $F_{1}$ is:

$$
\begin{gathered}
P_{I B \text { Swide }}=\sum_{s \in\{2,4,6\}} \sum_{b=1}^{19}\left[P_{B} \cdot G \cdot A_{T} \cdot A_{R}\right], \\
P_{I B \text { Snarrow }}=\sum_{s \in\{1,3,5\}} \sum_{b=1}^{19}\left[P_{B} \cdot G_{n} \cdot A_{I n} \cdot A_{R}\right]-P_{r},
\end{gathered}
$$

where $G=G\left(b, s, F_{1}, k\right)$ and $G_{n}=G_{n}\left(b, s, F_{1}, k\right)$ are the channel gains from wide and narrow beam of sector $s$ of $B S_{\mathrm{b}}$ to $F_{1}$, with $A_{T}=A_{T}\left(b, s, F_{1}\right)$ and $A_{T n}=A_{T n}\left(b, s, F_{1}\right)$ being the antenna gains, respectively. $A_{R}=A_{R}\left(b, s, F_{1}\right)$ is the receiving gain of FRS antenna.

User $d_{m}$ in set $\mathbf{D}$ is interfered by all BSs. The mean power of useful signal and interference from wide beams of BSs, i.e., $P_{r}$ and $P_{I B S w i d e}$, on sub-channel $k$ of user $d_{m}$, are the same as (1) and (2), respectively. The mean power of interference from the narrow beams of BSs is:

$$
P_{\text {IBSnarrow }}=\sum_{s \in\{2,4,6\}} \sum_{b=1}^{19}\left[P_{B} \cdot G_{n} \cdot A_{T n}\right],
$$

where $G_{n}=G_{n}\left(b, s, d_{m}, k\right)$ and $A_{T n}=A_{T n}\left(b, s, d_{m}\right)$ are the channel gain and antenna gain from the narrow beam of sector $s$ of $B S_{\mathrm{b}}$ to $d_{m}$, respectively.

In the SRS system, the number of relay stations is 27 in the 19-cell network, and each BS sector uses $N$ sub-channels. When variable $s$ in (2) and (5) is chosen from $\{1,2,3\}$, and $N_{r}$ in (3) and (6) is $27,(1)$ to (8) are just the corresponding formulations of the SRS system.

At last, the average CINR on sub-channel $k$ of user $u$ can be expressed as, 


$$
\operatorname{CINR}(u, k)=\frac{P_{r}}{P_{I}+N_{0} \cdot \Delta f},
$$

where $P_{r}$ and $P_{I}$ are the mean power of useful signal and the total interference, $N_{0}$ and $\Delta f$ are the AWGN power spectral density and subcarrier spacing, respectively.

\section{RATE MAPPING, PATH SELECTION AND SCHEDULING}

\section{A. Rate Mapping}

In multi-user OFDMA systems, the channel states of different users are fading independently, hence a channel that is in deep fading for user A may be a good channel for user $\mathrm{B}$. When adaptive modulation and coding (AMC) is adopted, better channel state yields higher data rate. If sub-channels are allocated to users with better channel states, then the greater the number of the users is, the higher the throughput is, known as multi-user diversity [10]. When the coding scheme is Convolutional Turbo Code (CTC) and the frame error rate is lower than $10 \%$, the relationship between CINR and the modulation and coding scheme (MCS) is listed in Table I [6].

If $\mathrm{M}$-ary $\left(\mathrm{M}=2^{p}\right)$ modulation and the coding with rate $a$ is used in an MCS, then the bits that one subcarrier in an OFDM symbol can carry is $p \cdot a$. Assume the channel is stable during a frame, when the mean CINR of a sub-channel is $\Gamma$, the corresponding achievable data rate is:

$$
\operatorname{rate}(\Gamma)=p \cdot a \cdot c \cdot N_{s} \cdot \frac{1}{T_{F}},
$$

where $T_{F}$ is the frame length, $c$ and $N_{s}$ are the number of sub-carriers in a sub-channel and the number of OFDM symbol in each frame, respectively.

\section{B. Path Selection Strategy}

In single hop cellular systems, MSs can only access to BSs, thus there is no need for path selection. However, in two hop networks, MSs can access to BSs or FRSs, thus path selection based on channel state is needed. In order to reduce the signaling overhead, the path selection can be carried out by MSs [11]. In the BBRS system, FRSs broadcast the mean CINR of each sub-channel on BS-FRS link, i.e., $\Gamma_{B F}$. According to the preamble of BS and FRS, MSs calculate the mean CINR of each sub-channel on BS-MS links and FRS-MS links, i.e., $\Gamma_{B M}$ and $\Gamma_{F M}$.

TABLE I.

RELATIONSHIP BETWEEN CINR AND MCS

\begin{tabular}{|c|c|c|c|}
\hline MCS & CINR (dB) & MCS & CINR (dB) \\
\hline QPSK (1/12) & -3.14 & 16QAM(1/2) & 9.94 \\
\hline QPSK (1/6) & -0.73 & 16QAM(2/3) & 13.45 \\
\hline QPSK (1/3) & 2.09 & 64QAM(2/3) & 18.6 \\
\hline QPSK (1/2) & 4.75 & 64QAM(5/6) & 24.58 \\
\hline QPSK (2/3) & 7.86 & & \\
\hline
\end{tabular}

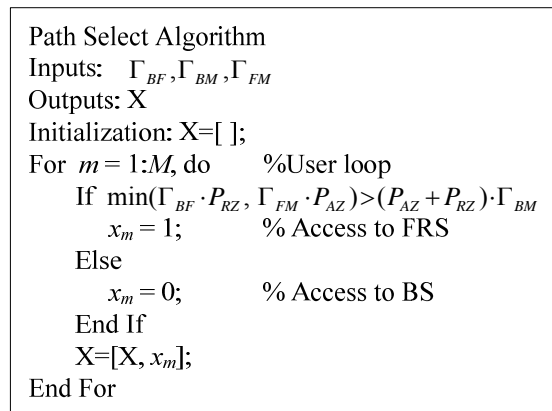

Figure 6. Path selection strategy.

Based on CINR values obtained and take the bottleneck effect of two hop communications into consideration [6], the pseudocode of the path select strategy can be described in Fig. 6, where $P_{A Z}$ and $P_{R Z}$ are the proportions of AZ and RZ to the frame length.

\section{Proportional Fair Scheduling}

To maintain low complexity, relay stations usually do not have the ability of scheduling, and all the scheduling is performed by base stations. Assume the access points of users do not change during a frame. Due to different interference states, users may have distinct achievable rates in $A Z$ and $R Z$. In order to evaluate the system performance more precisely, let BSs allocate resource once in $\mathrm{AZ}$ and $\mathrm{RZ}$, respectively. Consider the signaling overhead, BSs may execute scheduling once in a frame in practice. Proportional fair scheduling is considered in this paper, BSs assign a sub-channel to users with the highest priority at the beginning of each time zone. At time $t$, the priority of user $u$ on sub-channel $k$ is:

$$
\varphi(u, k, t)=\frac{r(u, k, t)}{\bar{R}(u, t-1)},
$$

where $r(u, k, t)$ is the instantaneous achievable data rate of user $u$ on sub-channel $k, \bar{R}(u, t)$ is the average obtained data rate in the latest time window with length $N_{W}$, up to time $t, u \in\{1: M\}$. Assume the obtained data rate of user $u$ at time $t$ is $R(u, t)$, then $\bar{R}(u, t)$ is updated according to:

$$
\bar{R}(u, t)=\left(1-\frac{1}{N_{W}}\right) \cdot \bar{R}(u, t-1)+\frac{1}{N_{W}} \cdot R(u, t) .
$$

At time $t, \mathrm{BS}$ assigns sub-channel $k$ to user:

$$
u^{*}=\arg \max _{u}[\varphi(u, k, t)]
$$

\section{1) Scheduling in BBRS System}

In the BBRS system, a BS sector uses two beams, with the same number of sub-channels, i.e., $N / 2$, to send data to direct users (users that access to BS) and FRS, thus certain fairness between direct users and relay users (users that access to FRS) is achieved already, and the side effect on the resource assignment to relay users is eliminated to some extent. Therefore, it is suitable to schedule direct users and relay users separately.

2) Scheduling in SRS System 
In the SRS system, a BS sector only has one beam layer, so direct users and the FRS need to share $N$ sub-channels. The amount of sub-channels that FRS attained in the $1^{\text {st }}$ hop has an important influence on the performance of direct users and relay users, and an appropriate priority value for the FRS is needed.

Consider sector $s_{1}$ of $B S_{1}$, which is the area with bold border in Fig. 3. Users that access to $B S_{1}$ and $F_{1}$ are $\mathbf{D}=\left\{d_{m}, m=1,2, \ldots, M_{1}\right\} \quad$ and $\quad \mathbf{Q}=\left\{q_{n}, n=1,2, \ldots, M_{2}\right\}$, respectively, with $M_{1}+M_{2}=M$. Assume the achievable data rates of users in $\mathbf{D}$ and $\mathbf{Q}$ are $r\left(d_{m}, k\right), m \in\left\{1: M_{1}\right\}$ and $r\left(q_{n}, k\right), n \in\left\{1: M_{2}\right\}$. The average data rate of relay station $F_{1}$ can be represented by:

$$
\bar{R}\left(F_{1}, t\right)=\frac{1}{|\mathbf{Q}|} \sum_{n=1}^{M_{2}} \bar{R}\left(q_{n}, t\right) .
$$

$F_{1}$ use sub-channel set $r_{3}$ to serve relay users, which are users in $\mathbf{Q}$. Sub-channel $k$ is allocated to the user:

$$
\hat{n}(k)=\arg \max _{n \in\left\{1: M_{2}\right\}}\left[\varphi\left(q_{n}, k\right)\right] .
$$

In order to fully exploit the spectrum resource in the $2^{\text {nd }}$ hop, the channel states of all the relay users must be taken into consideration [12]. Introduce a match factor,

$$
\beta=\frac{1}{R\left(F_{1}, t\right)} \cdot \sum_{k \in r_{3}} r\left(q_{\hat{n}(k)}, k\right),
$$

where $R\left(F_{1}, t\right)$ is the obtained data rate of $F_{1}$ at time $t$, and the $2^{\text {nd }}$ item on the right-hand side is the sum throughput of all relay users. $\beta$ is updated if $F_{1}$ get a sub-channel, and $F_{1}$ quits the competition when $\beta \leq 1$.

If the achievable data rate of $F_{1}$ on sub-channel $k$ is $r\left(F_{1}, k, t\right)$, then the priority of $F_{1}$ is,

$$
\varphi\left(F_{1}, k, t\right)=\beta \cdot \frac{r\left(F_{1}, k, t\right)}{\bar{R}\left(F_{1}, t-1\right)} .
$$

\section{3) Throughput}

Direct users can get services in the whole downlink sub-frame, while relay users can only be served in the downlink AZ. Thus the downlink throughput of Cell $_{1}$ can be expressed as,

$$
T_{D L}=\sum_{s=1}^{S_{T}}\left\{\begin{array}{l}
\sum_{m=1}^{M_{1}(s)}\left[P_{A Z} \cdot R_{A Z}\left(s, d_{m}\right)+P_{R Z} \cdot R_{R Z}\left(s, d_{m}\right)\right]+ \\
\sum_{n=1}^{M_{2}(s)} P_{A Z} \cdot R_{A Z}\left(s, q_{n}\right)
\end{array}\right\},
$$

where $S_{T}, P_{A Z}$ and $P_{R Z}$ are the number of sectors of Cell ${ }_{1}$, the proportions of $A Z$ and $R Z$ to the frame length, respectively. $M_{1}(s)$ and $M_{2}(s)$ are the number of direct users and relay users in sector $s$ of $\operatorname{Cell}_{1}, R_{A Z}(s, u)$ and $R_{R Z}(s, u)$ are the throughputs of user $u$ in $\mathrm{AZ}$ and $\mathrm{RZ}$, respectively.

\section{NUMERICAL RESULTS AND DISCUSSION}

\begin{tabular}{|c|c|c|}
\hline \multicolumn{2}{|r|}{ Parameters } & value \\
\hline \multicolumn{2}{|r|}{ Cell radius } & $1 \mathrm{~km}$ \\
\hline \multicolumn{2}{|c|}{ Central carrier freq./Bandwidth } & $2 \mathrm{GHz} / 5 \mathrm{MHz}$ \\
\hline \multicolumn{2}{|c|}{$c / N$} & $10 / 30$ \\
\hline \multicolumn{2}{|c|}{ Subcarrier spacing $\Delta f$} & $10.94 \mathrm{kHz}$ \\
\hline \multirow{2}{*}{ Sector power } & BBRS: BS/FRS high/low & $10 / 3.3 / 3.3 \rho \mathrm{W}$ \\
\hline & SRS: $\quad$ BS/FRS & $20 / 6.6 \mathrm{~W}$ \\
\hline \multicolumn{2}{|c|}{$G_{\mathrm{m}}: \mathrm{BS}$ (wide/narrow)/FRS } & $17 / 21 / 14 \mathrm{dBi}$ \\
\hline \multicolumn{2}{|c|}{$A_{\mathrm{m}}: \mathrm{BS}$ (wide/narrow)/FRS } & $23 / 27 / 20 \mathrm{dBi}$ \\
\hline \multicolumn{2}{|c|}{$\theta_{3 d B}: \mathrm{BS}$ (wide/narrow)/FRS } & $35 / 15 / 70$ deg. \\
\hline \multicolumn{2}{|c|}{ Number of OFDM symbols in a frame } & 47 \\
\hline \multicolumn{2}{|c|}{ Length of DL sub-frame/UL sub-frame } & $5 / 3$ \\
\hline \multicolumn{2}{|c|}{ Length of DL AZ/ DL RZ } & $1 / 1$ \\
\hline \multicolumn{2}{|c|}{ Pathloss index (LOS/NLOS) } & $2.35 / 3.76$ \\
\hline \multicolumn{2}{|c|}{ Std. deviation of shadowing (LOS/NLOS) } & $3 / 8 \mathrm{~dB}$ \\
\hline \multicolumn{2}{|r|}{ User velocity } & $3 \mathrm{~km} / \mathrm{h}$ \\
\hline
\end{tabular}

In This section, the performance of the BBRS and SRS system is compared, and main simulation parameters are
TABLE II.

SIMULATION PARAMETERS

listed in table II. Monte Carlo simulation is performed, and all the results are averaged across 1000 drops. Since the number of sectors of each cell of two systems are 6 and 3, respectively, so the number of users in each BS sector in SRS system is set to two times of that in BBRS system to keep the same load.

\section{A. Channel Model}

Distance based pathloss, lognormal shadowing and small scale fading are taken into consideration.

When carrier frequency is $2 \mathrm{GHz}$, the pathloss is [13]:

$$
P L=128.1+\alpha \cdot 10 \cdot \lg (R)(d B),
$$

where $R$ (in $\mathrm{km}$ ) is the distance between source and destination, and $\alpha$ is the pathloss index.

The shadowing effect can be modeled as a variable with normal distribution, $S=N(0, \sigma)(d B)$.

The channels between BS and FRSs in the same cell are considered as in line of sight (LOS) environment, while all the other channels in the system are considered as in non-line of sight (NLOS) environment. The fading under LOS and NLOS can be modeled as Rician and Rayleigh fading, respectively. Based on the SUI-1 [14] and ITU-Pedestrian A channel model [15], employ the sum-of-sinusoids simulation model proposed in [16], the two types of fading can be expressed as follows,

$$
\begin{gathered}
Y(t)=\frac{1}{\sqrt{N_{p}}} \sum_{n=1}^{N_{p}} \exp \left[j\left(\omega_{d} t \cos \alpha_{n}+\phi_{n}\right)\right], \\
Z(t)=\sqrt{\frac{K}{1+K}} \exp \left[j\left(\omega_{d} t \cos \theta_{0}+\phi_{0}\right)\right]+ \\
\sqrt{\frac{1}{1+K}} \frac{1}{\sqrt{N_{p}}} \sum_{n=1}^{N_{p}} \exp \left[j\left(\omega_{d} t \cos \alpha_{n}+\phi_{n}\right)\right],
\end{gathered}
$$

where $N_{p}, \omega_{d}$, and $K$ are the number of propagation paths, the maximum radian Doppler frequency and Rician factor, respectively. $\alpha_{n}=\left(2 \pi n+\theta_{n}\right) / N_{p}, n=1,2, \ldots, N_{p}$ and $\phi_{n}$ 
are the angle of arrival and initial phase of the $n^{\text {th }}$ propagation path, $\theta_{0}$ and $\phi_{0}$ are the angle of arrival and the initial phase of the specular component, respectively. $\phi_{n}, \theta_{n}$ and $\phi_{0}$ are random variables uniformly distributed over $[-\pi, \pi)$.

Assume the small scale fading is $\zeta(d B)$, and the channel gain is defined by:

$$
G=10^{0.1(-P L+S+\zeta)} \text {. }
$$

All MSs use omnidirectional antennas with $0 d B$ gain. BSs and FRSs are equipped with sector antennas with the directional gain model as follows [14],

$$
A(\theta)=10^{0.1\left\{G_{m}-\min \left[12 \cdot\left(\frac{\theta}{\theta_{3 d B}}\right)^{2}, A_{m}\right]\right\}},
$$

where $G_{m}$ and $A_{m}$ are the maximum gain and the maximum attenuation (also known as the front-to-back ratio), $\theta_{3 d B}$ is the $3 d B$ beam width, and $\theta$ is the angle between the maximum gain direction of the antenna and the target, respectively.

Assume the transmit power of subcarrier is $P$, and the power of received signal will be,

$$
P_{r}=P \cdot G \cdot A_{T}\left(\theta_{T}\right) \cdot A_{R}\left(\theta_{R}\right),
$$

where $A_{T}\left(\theta_{T}\right)$ and $A_{R}\left(\theta_{R}\right)$ are the transmit and receive antenna gains with $\theta_{T} / \theta_{R}$ being the angle between the maximum gain direction of the transmitter/receiver antenna and the receiver/transmitter, respectively.

\section{B. CINR Distribution}

The numbers of users in each BS sector, i.e., $M$, in the SRS and BBRS systems, are set to 600 and 300 , respectively. Only the pathloss is considered. The MCSs corresponding to the mean CINR across all available sub-channels of all users in the central cell in AZ are recorded. Seven MCSs considered are outage $(\mathrm{CINR}<-3.14 d B)$ QPSK $(1 / 12)$ to $\operatorname{QPSK}(1 / 2)$, QPSK(2/3), 16QAM(1/2), 16QAM(2/3), 64QAM(2/3) and 64QAM(5/6), numbered from 1 to 7 , respectively.

Fig. 7 illustrates that, compared with the SRS system, the outage area is decreased sharply, and better coverage performance is achieved in the BBRS system. Co-channel interference is suppressed by the dedicated cell architecture and frequency planning, thus higher MCSs can be used by direct users.

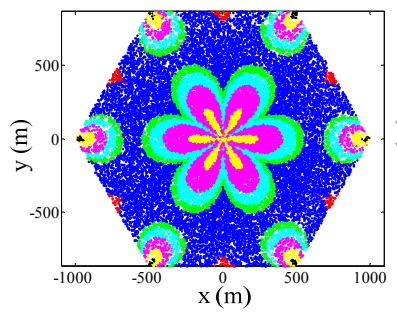

(a)

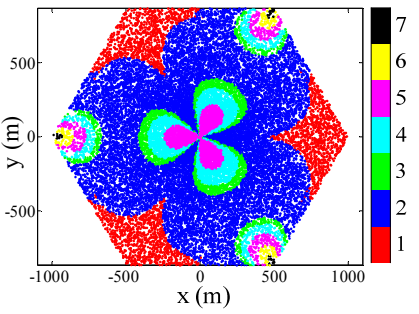

(b)
Figure 7. CINR distribution in central cell of BBRS (a) and SRS (b).

\section{Multi-user Performance}

A multi-user performance metric of relay cellular system, i.e., the combined coverage and capacity index (cc), is defined by IEEE $802.16 \mathrm{j}$ working group [14]:

$$
C C=\frac{k}{\sum_{j=1}^{k} \frac{R_{\min }}{r_{j}}},
$$

where $r_{j}$ is the obtained data rate of user $j$, and $R_{\min }$ is the minimum data rate requirement.

If the total number of users is large, then $c c$ approaches the expected value of the number of users that can be supported by the system. The $c c$ curve under power ratio $\rho=0.5$ and $80 \%$ coverage requirement is shown as Fig. 8 . We can see that the performance of the BBRS system is better than the SRS system, when $R_{\min }$ changing from $10 \mathrm{kbits} / \mathrm{s}$ to $200 \mathrm{kbits} / \mathrm{s}$.

\section{Influence of Non-ideal Characteristics of Antennas}

The non-ideal characteristics of the sector antennas have significant influence on system throughput. Raise the maximum attenuation, i.e., $A_{m}$, of the two systems in step of $3 d B$ for 4 times, the same analysis is conducted for the maximum gain $G_{m}$ as well. The throughput performance is shown as Fig. 9. With the increasing of $A_{m}$, the interference to other directions is reduced, and the throughputs of two systems both rise. However, the influence of $G_{m}$ is quite different from $A_{m}$. In the SRS system, the raise of $G_{m}$ reduces the proportion of the outage area, achieving a higher throughput. But in the BBRS system, a higher coverage percentage has already achieved, the raise of $G_{m}$ causes greater co-channel interference, resulting in lower throughput.

\section{E. Influence of Frequency Reuse}

Soft frequency reuse is considered in the proposed BBRS system. The coverage of the FRSs and system throughput will be affected if the power ratio $\rho$ is changed. When the total number of users in Cell $_{1}$ is set to $60,120,180,240$ and 300, and the power ratio $\rho$ is set to $0,0.25$ and 1 , the throughput performance of Cell 1 is shown as Fig. 10. Due to the multi-user diversity, the

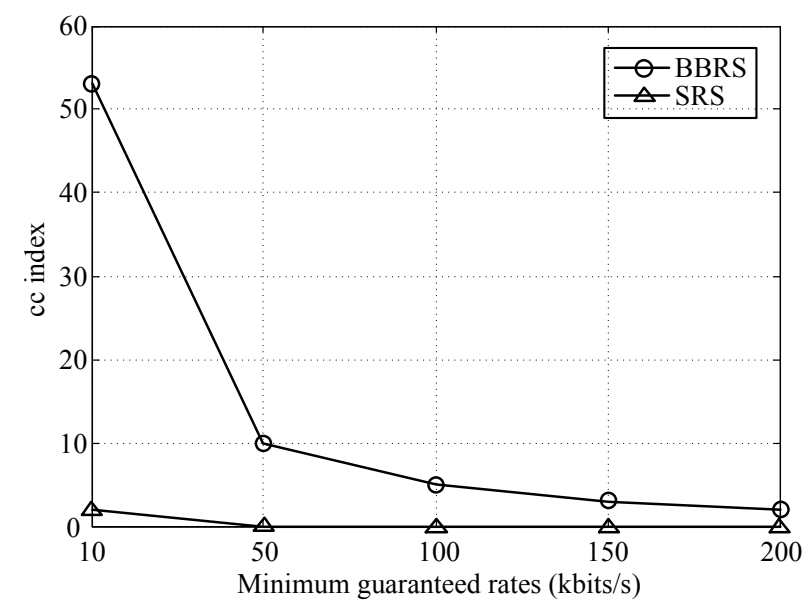

Figure 8. cc performance. 


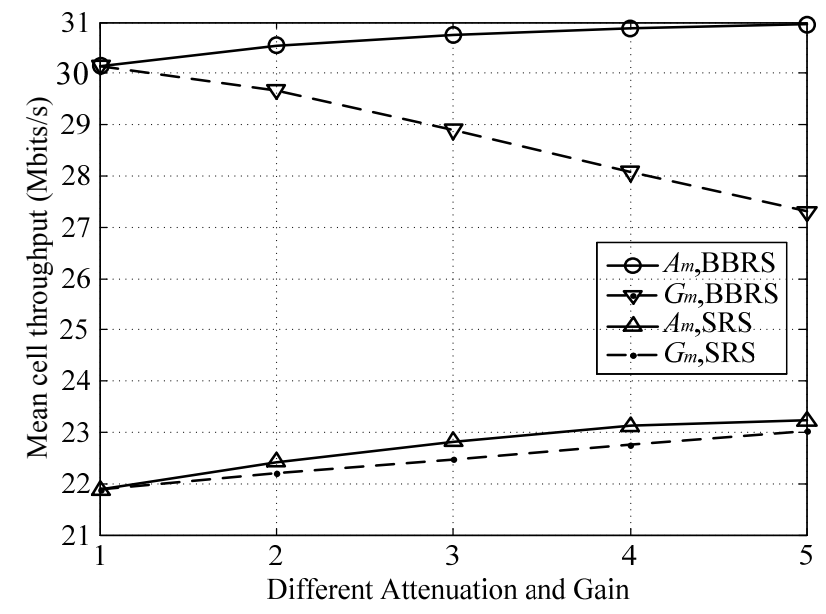

Figure 9. Influence of antenna parameters to throughput.

bigger the number of users is, the higher the throughput is. The growth of the throughput slows down with the increasing of the power ratio. A good compromise is achieved between the throughput and the power consumption when $\rho=0.25$, compared with $\rho=0$, i.e., the scenario with no soft frequency reuse on FRSs.

The available number of sub-channels for each BS sector in the BBRS system is only one half of that in the SRS system, which may result in lower throughput. However, due to the effectiveness of interference mitigation, higher throughput can be expected. It can be seen clearly from Fig. 10 that, the performance improvement of the BBRS system is noticeable. There is an increase about $27 \% \sim 34 \%$ over the SRS system, even though no soft frequency reuse on FRSs is considered.

The results upon imply that more available sub-channels do not represent higher throughput, for the co-channel interference has an important influence on the performance. When there are no relay stations, the co-channel interference is reduced further. The throughputs of the SRS and BBRS systems raise about $11 \%$ to $12 \%$ and decrease about $11 \%$ to $19 \%$. However, the latter is still higher than the former, which demonstrates that the frequency planning performance of the manner with orthogonal sub-channels assigned to adjacent sectors in the same cell is better than that of the

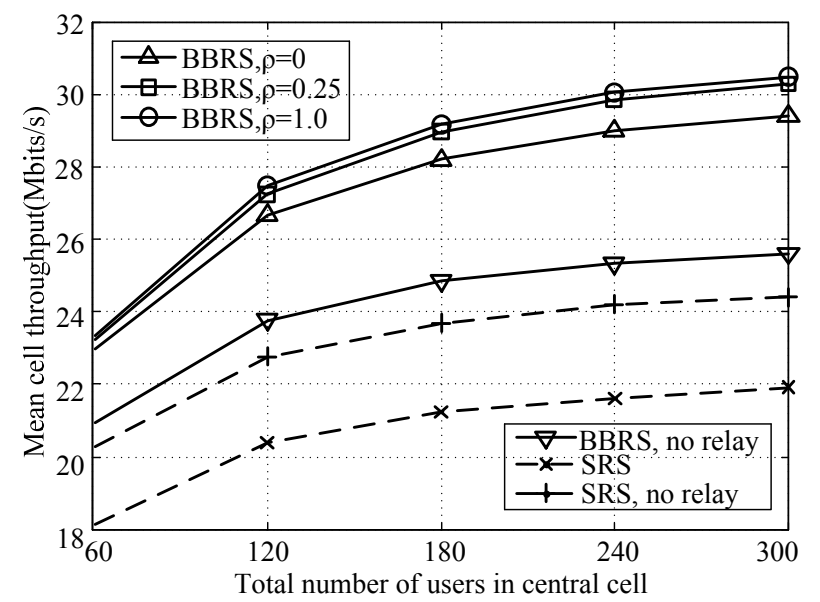

Figure 10. Central cell throughput comparison.

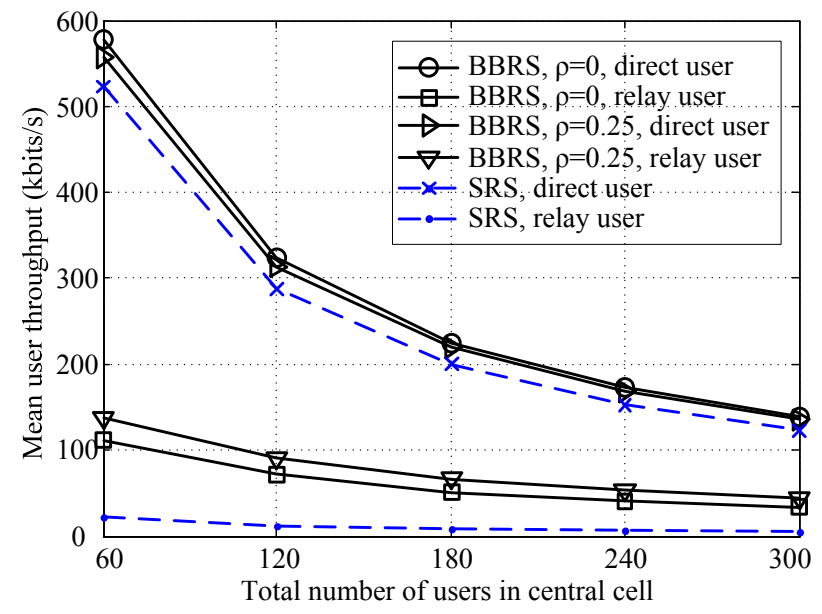

Figure 11. Mean throughput of direct users and relay users.

aggressive frequency reuse in which all sectors using the whole system band.

\section{F. The Performance of Direct Users and Relay Users}

One of the main roles of relay stations in relay cellular systems is to improve the data rates of cell edge users. As a relay network, cell edge performance of the proposed BBRS system needs to be evaluated.

As shown in Fig. 11, compared with the SRS system, in the BBRS system, when $\rho=0.25$, not only does the average data rate of relay users increase by five to eight times but also that of direct users rises slightly. Furthermore, compared with $\rho=0$, when $\rho=0.25$, due to more serious intra-cell interference, the performance of direct users deceases, but that of relay users increases, for more sub-channels is available. Therefore, a compromise between the performance of direct users and relay users needs to be considered when choosing the parameter $\rho$.

\section{CONCLUSION}

In this paper, a bilayer beams and relay sharing based (BBRS) OFDMA cellular architecture and corresponding frequency planning scheme are proposed. From the simulation results we can conclude that, sector antennas can suppress interference in OFDMA cellular systems efficiently, and the aggressive frequency reuse without considering the co-channel interference will result in lower system performance.

Due to the spectrum resource is limited, the growing demand for higher data rate makes it necessary to consider tighter frequency reuse, where co-channel interference is more serious and a higher requirement for interference suppression is needed. In order to solve this problem, several techniques can be considered. Firstly, in the proposed BBRS system, the shared relay stations provide a convenient way for local interference coordination, in which the main interference can avoid and a substantial performance improvement can be expected. Secondly, cooperation between base stations and relay stations may improve cell edge users' performance further. Thirdly, a better performance can be expected if dynamic frequency planning is employed when cell load changes fast. All the 
aspects mentioned above will be included in our future work.

\section{ACKNOWLEDGMENT}

The authors wish to thank the anonymous reviewers for their constructive comments that have improved the quality of this paper.

\section{REFERENCES}

[1] 3GPP, "TR 25.814 V7.1.0, Physical layer aspects for E-UTRA," 2006.

[2] "IEEE Standard for Local and metropolitan area networks Part 16: Air Interface for Broadband Wireless Access Systems Amendment 1: Multiple Relay Specification," IEEE Std 802.16j-2009 (Amendment to IEEE Std 802.16-2009), pp. c1-c290, 2009.

[3] Loa K., Chih-Chiang Wu, Shiann-Tsong Sheu, Yifei Yuan, Chion M. and Huo D., et al., "IMT-advanced relay standards [WiMAX/LTE Update]," IEEE Commun. Mag., vol.48, pp. 40-48, 2010.

[4] Boudreau G., Panicker J., Ning Guo, Rui Chang, Neng Wang and Vrzic S., "Interference coordination and cancellation for 4G networks," IEEE Commun. Mag., vol.47, pp. 74-81, 2009.

[5] Yushan Pei, Hui Tian, Tong Wu and Ying Wang, "Frequency Planning Scheme Based on Interference Coordination for OFDM-Relay Systems," IEEE Commun. Letters, vol.15, pp. 13-15, 2011.

[6] Zhengguang Zhao, Xuming Fang, Ying Zhu and Yan Long, "Two Frequency Reuse Schemes in OFDMA-TDD Based Two-Hop Relay Networks," in Proc. IEEE WCNC 2010, pp. 1-6.

[7] Lin Qu, Xiaoxiang Wang, Yulong Wang and Jianxin Liao, "Performance Evaluation of Frequency Planning in a Novel Cellular Architecture Based on Sector Relay," in Proc. IEEE VTC 2010-Spring, pp. 1-5.

[8] Lee J. and Yanikomeroglu H., "A Novel Architecture for Multi-Hop WiMAX Systems: Shared Relay Segmentation," in Proc. IEEE WCNC 2010, pp. 1-6.

[9] Min Liang, Fang Liu, Zhe Chen, Ya Feng Wang and Da Cheng Yang, "A Novel Frequency Reuse Scheme for OFDMA Based Relay Enhanced Cellular Networks," in Proc. IEEE VTC 2009-Spring, pp. 1-5.

[10] Rahman M. and Yanikomeroglu H., "Enhancing cell-edge performance: a downlink dynamic interference avoidance scheme with inter-cell coordination," IEEE Trans. Wireless Commun., vol.9, pp. 1414-1425, 2010

[11] Park W. H. and Bahk S., "Resource management policies for fixed relays in cellular networks," Computer Communications, vol.32, pp. 703-711, 2009.

[12] Lin Xiao and Cuthbert L., "A Two-Hop Proportional Fairness Scheduling Algorithm for Relay Based OFDMA Systems," in Proc. IEEE WiCOM 2008, pp. 1-4.

[13] Haipeng Lei, Lei Zhang, Xin Zhang and Dacheng Yang, "A Novel Multi-Cell OFDMA System Structure using Fractional Frequency Reuse," in Proc. IEEE PIMRC 2007, pp. 1-5.

[14] Senarath G., Tong W. and Zhu P., "Multi-hop Relay System Evaluation Methodology (Channel Model and Performance Metric)," IEEE C802.16j-06/013r3, 2007.

[15] ITU, Guidelines for Evaluation of Radio Transmission Technologies for IMT-2000, 1997.

[16] Chengshan Xiao, Yahong Rosa Zheng and Beaulieu N. C., "Novel Sum-of-Sinusoids Simulation Models for Rayleigh and Rician Fading Channels," IEEE Trans. Wireless Commun., vol.5, pp. 3667-3679, 2006.

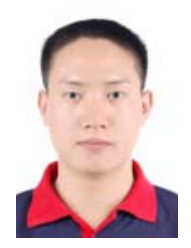

Yanxiong Pan was born in 1982. He received his B.S. degree in Space Tracking Telemetry and Command from Academy of Equipment Command \& Technology of P.L.A., Beijing, China, in 2004. Since 2008, he has been working toward the M.S. degree in Electrical Engineering at University of Science and Technology of China, Hefei, China.

$\mathrm{He}$ was an engineer at Yibin Tracking Telemetry Station, Xichang Satellite Launch Center, Yibin, China, from 2004 to 2008. His current research interests include radio resource management and relay communication.

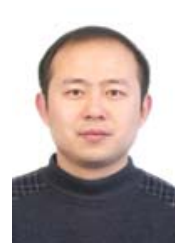

Hui Han received the B.S. degree from Chongqing Communication Institute of P.L.A., in 2002, and the M.S. degree in Electrical Engineering from University of Science and Technology of China, Hefei, China, in 2009.

$\mathrm{He}$ is currently a lecturer in the department of Information Engineering, Chongqing Communication Institute of P.L.A. His research interests include error control coding and wireless communication.

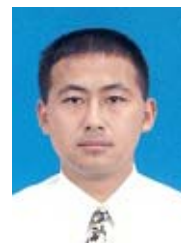

Sihai Zhang received the B.S. degree in Computer Science (CS) from Ocean University of China, in 1996, and the M.S. and Ph.D. degrees in CS from University of Science and Technology of China (USTC), Hefei, China, in 2002 and 2006, respectively.

$\mathrm{He}$ is currently a lecturer in the department of Electronic Engineering and Information Science, USTC. His research interests include evolutionary computation, wireless communication network, and social network.

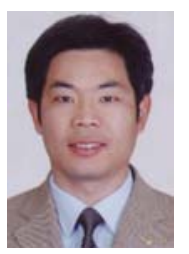

Wuyang Zhou received his B.S. and M.S. degrees from Xidian University, Xi'an, China, in 1993 and 1996, respectively, and Ph.D. degree in Electrical Engineering from University of Science and Technology of China (USTC), Hefei, China, in 2000.

$\mathrm{He}$ is currently a professor in the department of Electronic Engineering and Information Science, USTC. His research interests include error control coding, radio resource allocation, and wireless networking. 